\title{
Neutral Gauge Boson Contributions to the Dimuon Charge Asymmetry in $B$ Decays
}

\author{
Ashutosh Kumar Alok ${ }^{a}$, Seungwon Baek ${ }^{b}$, David London ${ }^{a}$ \\ a Physique des Particules, Université de Montréal, \\ C.P. 6128, succ. centre-ville, Montréal, QC, Canada H3C 3J7 \\ ${ }^{b}$ The Institute of Basic Science and Department of Physics, Korea University, \\ Seoul 136-701, Republic of Korea \\ E-mail: alok@lps.umontreal.ca, sbaek@korea.ac.kr, \\ london@lps.umontreal.ca.
}

\begin{abstract}
Recently, the DØ Collaboration measured the CP-violating like-sign dimuon charge asymmetry in neutral $B$ decays, finding a $3.2 \sigma$ difference from the standard-model (SM) prediction. A non-SM charge asymmetry $a_{\mathrm{sl}}^{s}$ suggests a newphysics (NP) contribution to $B_{s}-\bar{B}_{s}$ mixing. In this case, in order to explain the measured value of $a_{\mathrm{sl}}^{s}$ within its $1 \sigma$ range, NP must be present in $\Gamma_{12}^{s}$, the absorptive part of the mixing. In this paper, we examine whether such an explanation is possible in models with flavor-changing $Z$ ( $Z \mathrm{FCNC})$ or $Z^{\prime}\left(Z^{\prime} \mathrm{FCNC}\right)$ gauge bosons. The models must also reproduce the measured values of the indirect CP asymmetry $S_{\psi \phi}$ in $B_{s} \rightarrow J / \psi \phi$, and $\Delta \Gamma_{s}$, the $B_{s}-\bar{B}_{s}$ width difference. We find that the $Z \mathrm{FCNC}$ model cannot reproduce the present measured values of $S_{\psi \phi}$ and $a_{\mathrm{sl}}^{s}$ within their $1 \sigma$ ranges. On the other hand, in the $Z^{\prime}$ FCNC model, the values of all three observables can be simultaneously reproduced.
\end{abstract}

KEywords: Flavor-Changing Gauge Boson, $B_{s}-\bar{B}_{s}$ mixing, Beyond Standard Model. 


\section{Contents}

1. Introduction 1

2. Contributions to $\Gamma_{12}^{s}$

3. New-Physics Models 6

$3.1 \quad Z$-mediated FCNC's 6

$3.2 \quad Z^{\prime}$-mediated FCNC's

4. Numerical Results 9

$4.1 \quad Z$ FCNC 9

$4.2 \quad Z^{\prime} \mathrm{FCNC}$

4.2.1 $\mathrm{NP} b \rightarrow s \tau^{+} \boldsymbol{\tau}^{-}$operator only

$4.2 .2 \mathrm{NP} b \rightarrow s c \bar{c}$ operator only 12

5. Conclusions 14

\section{Introduction}

Flavor-changing neutral-current (FCNC) processes occur only at the loop level in the standard model (SM), and are therefore a very sensitive probe of physics beyond the SM. Interestingly, recently there have been several measurements of quantities in $B$ decays, especially in the $b \rightarrow s$ transition, which differ from the predictions of the SM. For example, i) the value of $\sin 2 \beta$ measured in several $b \rightarrow s$ penguin decays is found to be smaller than that obtained in $B_{d} \rightarrow J / \psi K_{S}$ [囯], ii) in $B \rightarrow \pi K$, the SM has some difficulty in accounting for all the experimental measurements [2], iii) the forward-backward asymmetry in $B \rightarrow K^{*} \mu^{+} \mu^{-}$shows a small deviation from the SM, especially at low momentum transfer squared [3], iv) the measurement of CP violation in $B_{s} \rightarrow J / \psi \phi$ suggests the presence of a new-physics (NP) CP-violating phase in $B_{s}-\bar{B}_{s}$ mixing $[4]$. Although these effects are not significant enough to claim $\mathrm{NP}$, at least they indicate that flavor physics will still play a very important role in the search for NP in the LHC era. The LHCb will provide much more precise data on the above-mentioned observables.

Recently the DØ Collaboration reported an anomalously large CP-violating likesign dimuon charge asymmetry in the $B$ system [5]. The asymmetry is

$$
A_{\mathrm{sl}}^{b} \equiv \frac{N_{b}^{++}-N_{b}^{--}}{N_{b}^{++}+N_{b}^{--}},
$$


where $N_{b}^{ \pm \pm}$is the number of events of $b \bar{b} \rightarrow \mu^{ \pm} \mu^{ \pm} X$. From the analysis of $6.1 \mathrm{fb}^{-1}$ of data, $\mathrm{D} \varnothing$ found

$$
A_{\mathrm{sl}}^{b}=-(9.57 \pm 2.51 \pm 1.46) \times 10^{-3}
$$

where the first (second) error is statistical (systematic). This exhibits a $3.2 \sigma$ deviation from the SM prediction, $A_{\mathrm{sl}}^{b, \mathrm{SM}}=\left(-2.3_{-0.6}^{+0.5}\right) \times 10^{-4}[6]$. The asymmetry can be written as a linear combination of $B_{d}$ and $B_{s}$ contributions [7]

$$
A_{\mathrm{sl}}^{b}=(0.506 \pm 0.043) a_{\mathrm{sl}}^{d}+(0.494 \pm 0.043) a_{\mathrm{sl}}^{s}
$$

where the semileptonic "wrong-charge" asymmetry $a_{\mathrm{sl}}^{q}(q=d, s)$ is given by

$$
a_{\mathrm{sl}}^{q}=\frac{\Gamma\left(\bar{B}_{q} \rightarrow \mu^{+} X\right)-\Gamma\left(B_{q} \rightarrow \mu^{-} X\right)}{\Gamma\left(\bar{B}_{q} \rightarrow \mu^{+} X\right)+\Gamma\left(B_{q} \rightarrow \mu^{-} X\right)} .
$$

Thus, the discrepancy may indicate a NP contribution to $B_{d^{-}} \bar{B}_{d}$ mixing and/or $B_{s}-\bar{B}_{s}$ mixing [7].

Using the current experimental value of $a_{\mathrm{sl}}^{d, \exp }=-0.0047 \pm 0.0046$ [5, 8] and the $\mathrm{SM}$ prediction $a_{\mathrm{sl}}^{d, \mathrm{SM}}=\left(-4.8_{-1.2}^{+1.0}\right) \times 10^{-4}$, Eq. (1.3) leads to $a_{\mathrm{sl}}^{s}=-0.0146 \pm 0.0075$. The CDF Collaboration also measured $A_{\mathrm{sl}}^{b}$, but with much larger errors: $A_{\mathrm{sl}}^{b}=$ $(8.0 \pm 9.0 \pm 6.8) \times 10^{-3}[9]$. Finally, DØ directly measured $a_{\mathrm{sl}}^{s}: a_{\mathrm{sl}}^{s, \exp }=-(1.7 \pm$ $\left.9.1_{-1.5}^{+1.4}\right) \times 10^{-3}$ [10]. Combining all these results, we get

$$
\left(a_{\mathrm{sl}}^{s}\right)_{\mathrm{ave}}=-(12.7 \pm 5.0) \times 10^{-3},
$$

which is still about $2.5 \sigma$ away from the SM prediction of $a_{\mathrm{sl}}^{s, \mathrm{SM}}=(2.1 \pm 0.6) \times 10^{-5}$.

The D $\varnothing$ measurement thus suggests the presence of NP in $B_{s}-\bar{B}_{s}$ mixing, and we explore this possibility below. We begin with a general review of the mixing. In the $B_{s}$ system, the mass eigenstates $B_{L}$ and $B_{H}$ ( $L$ and $H$ indicate the light and heavy states, respectively) are admixtures of the flavor eigenstates $B_{s}$ and $\bar{B}_{s}$ :

$$
\begin{aligned}
& \left|B_{L}\right\rangle=p\left|B_{s}\right\rangle+q\left|\bar{B}_{s}\right\rangle, \\
& \left|B_{H}\right\rangle=p\left|B_{s}\right\rangle-q\left|\bar{B}_{s}\right\rangle
\end{aligned}
$$

with $|p|^{2}+|q|^{2}=1$. As a result, the initial flavor eigenstates oscillate into one another according to the Schrödinger equation

$$
i \frac{d}{d t}\left(\begin{array}{l}
\left|B_{s}(t)\right\rangle \\
\left|\bar{B}_{s}(t)\right\rangle
\end{array}\right)=\left(M^{s}-i \frac{\Gamma^{s}}{2}\right)\left(\begin{array}{l}
\left|B_{s}(t)\right\rangle \\
\left|\bar{B}_{s}(t)\right\rangle
\end{array}\right)
$$

where $M=M^{\dagger}$ and $\Gamma=\Gamma^{\dagger}$ correspond respectively to the dispersive and absorptive parts of the mass matrix. The off-diagonal elements, $M_{12}^{s}=M_{21}^{s *}$ and $\Gamma_{12}^{s}=\Gamma_{21}^{s *}$, are generated by $B_{s}-\bar{B}_{s}$ mixing. We define

$$
\Gamma_{s} \equiv \frac{\Gamma_{H}+\Gamma_{L}}{2}, \quad \Delta M_{s} \equiv M_{H}-M_{L}, \quad \Delta \Gamma_{s} \equiv \Gamma_{L}-\Gamma_{H}
$$


Expanding the mass eigenstates and $q / p$ in $\Gamma_{12}^{s} / M_{12}^{s}$, we find, to a very good approximation [11,

$$
\begin{aligned}
\Delta M_{s} & =2\left|M_{12}^{s}\right| \\
\Delta \Gamma_{s} & =2\left|\Gamma_{12}^{s}\right| \cos \phi_{s} \\
\frac{q}{p} & =-e^{-i \phi_{M}^{s}}\left(1-\frac{\left|M_{12}^{s}\right|}{2\left|\Gamma_{12}^{s}\right|} \sin \phi_{s}\right),
\end{aligned}
$$

where $\phi_{M}^{s} \equiv \arg M_{12}^{s}$ and $\phi_{s} \equiv \arg \left(-M_{12}^{s} / \Gamma_{12}^{s}\right)$. Then $a_{\mathrm{sl}}^{s}$ is given by [6]

$$
a_{\mathrm{sl}}^{s}=\operatorname{Im}\left(\frac{\Gamma_{12}^{s}}{M_{12}^{s}}\right)=\frac{\left|\Gamma_{12}^{s}\right|}{\left|M_{12}^{s}\right|} \sin \phi_{s} \text {. }
$$

In the SM, we have

$$
a_{\mathrm{sl}}^{s}=(4.97 \pm 0.94) \times 10^{-3} \sin \phi_{s} .
$$

Within the SM, the phase of $M_{12}^{s}$ is given by $\arg \left[\left(V_{t b} V_{t s}^{*}\right)^{2}\right]$ and that of $\Gamma_{12}^{s}$ is dominated by $\arg \left[\left(V_{c b} V_{c s}^{*}\right)^{2}\right]$. The state-of-the-art calculation gives $\phi_{s}=0.0041 \pm 0.0008$ [6]. As noted above, the SM cannot explain the D $\varnothing$ result; this is due to its far-too-small weak phases. From here on, we neglect the SM weak phases.

Suppose now that NP contributes to $M_{12}^{s}$ but not to $\Gamma_{12}^{s}$. We introduce a new parameter $\Delta_{s}$ to take this into account: $M_{12}^{s}=M_{12}^{s, \mathrm{SM}}+M_{12}^{s, \mathrm{NP}}=M_{12}^{s, \mathrm{SM}} \Delta_{s}=$ $M_{12}^{s, \mathrm{SM}}\left|\Delta_{s}\right| e^{i \phi_{M}^{s}}$. We have

$$
a_{\mathrm{sl}}^{s}=\frac{\left|\Gamma_{12}^{s}\right|}{\left|M_{12}^{s, \mathrm{SM}}\right|} \frac{\sin \phi_{M}^{s}}{\left|\Delta_{s}\right|}=(4.97 \pm 0.94) \times 10^{-3} \frac{\sin \phi_{M}^{s}}{\left|\Delta_{s}\right|} .
$$

The experimental value of the mass difference in the $B_{s}-\bar{B}_{s}$ system, $\Delta M_{s}=(17.77 \pm$ $0.12) \mathrm{ps}^{-1}$, constrains $\left|\Delta_{s}\right|=0.92 \pm 0.32$. Then Eqs. (1.5) and (1.12) give $\sin \phi_{M}^{s}=$ $-2.56 \pm 1.16$, implying that the full $1 \sigma$ range of the experimental result lies outside the physical region. It is therefore not possible to explain the $1 \sigma$ range of the $\mathrm{D} \varnothing$ measurement if NP contributes only to $M_{12}^{s}$.

This problem can be solved if the new physics contributes to $\Gamma_{12}^{s}$. Here we introduce a second new parameter $\Xi_{s}: \Gamma_{12}^{s}=\Gamma_{12}^{s, \mathrm{SM}}+\Gamma_{12}^{s, \mathrm{NP}}=\Gamma_{12}^{s, \mathrm{SM}} \Xi_{s}=\Gamma_{12}^{s, \mathrm{SM}}\left|\Xi_{s}\right| e^{i \phi_{\Gamma}^{s}}$. We then have

$$
\begin{aligned}
a_{\mathrm{sl}}^{s} & =\frac{\left|\Gamma_{12}^{s, \mathrm{SM}}\right|}{\left|M_{12}^{s, \mathrm{SM}}\right|} \frac{\left|\Xi_{s}\right|}{\left|\Delta_{s}\right|} \sin \left(\phi_{M}^{s}-\phi_{\Gamma}^{s}\right) \\
& =(4.97 \pm 0.94) \times 10^{-3} \frac{\left|\Xi_{s}\right|}{\left|\Delta_{s}\right|} \sin \left(\phi_{M}^{s}-\phi_{\Gamma}^{s}\right) .
\end{aligned}
$$

Now Eq. (1.5) can be reproduced if $\left|\Xi_{s}\right|$ is sufficiently large.

The difficulty is that, in many NP models, the contribution to $\Gamma_{12}^{s}$ is not large enough to compete with that of the SM, which is dominated by the tree-level $b \rightarrow$ 
$s c \bar{c}$ process. Two exceptions are R-parity-violating supersymmetric models [12 and leptoquark models [13] (other analyses of Eq. (1.5) in various NP models can be found in Refs. [7, 14]).

In this paper we examine the contribution of flavor-changing neutral gauge bosons to $\Gamma_{12}^{s}$. We consider two types of models. They involve tree-level $Z \bar{b} s$ or $Z^{\prime} \bar{b} s$ couplings. As we will see, the non-universal $Z^{\prime}$ model can enhance $\Gamma_{12}^{s}$ enough to explain the $1 \sigma$ range of Eq. (1.5).

The paper is organised as follows. The NP contribution to $\Gamma_{12}^{s}$ is discussed in Sec. 2. In Sec. 3, we describe the models with flavor-changing $Z$ and $Z^{\prime}$ couplings. The numerical results are presented in Sec. 4. We conclude in Sec. 5.

\section{Contributions to $\Gamma_{12}^{s}$}

In the SM, the dominant contribution to $\Gamma_{12}^{s, \mathrm{SM}}$ comes from the charged-current $b \rightarrow$ $s c \bar{c}$ operator with a $c$-quark loop. It is given by

$$
\begin{aligned}
\Gamma_{12}^{s, \mathrm{SM}} & =-\frac{G_{F}^{2} m_{b}^{2} \lambda_{c}^{2}}{3 \pi} \sqrt{1-4 x_{c}} \times \\
& \left\{\left[K_{1}\left(1-x_{c}\right)+\frac{1}{2} K_{2}\left(1-4 x_{c}\right)\right]\left\langle O_{L L}\right\rangle+\left(K_{1}-K_{2}\right)\left(1+2 x_{c}\right)\left\langle\widetilde{O}_{R R}\right\rangle\right\},
\end{aligned}
$$

where $\lambda_{c}=V_{c b} V_{c s}^{*}, x_{c}=m_{c}^{2} / m_{b}^{2}$, and $K_{1}=3 C_{1}^{2}+2 C_{1} C_{2}, K_{2}=C_{2}^{2}$. The values for the $C_{i}$ are $C_{1}\left(m_{b}\right)=1.086, C_{2}\left(m_{b}\right)=-0.197$ (Tables 4,5 of Ref. [15]). We use the vacuum insertion approximation to calculate the hadronic matrix elements [16:

$$
\begin{aligned}
\left\langle O_{L L}\right\rangle & \equiv\left\langle B_{s}\left|\bar{s} \gamma_{\mu} P_{L} b \bar{s} \gamma^{\mu} P_{L} b\right| \bar{B}_{s}\right\rangle=\frac{1}{3} m_{B_{s}} f_{B_{s}}^{2}, \\
\left\langle\widetilde{O}_{R R}\right\rangle \equiv\left\langle B_{s}\left|\bar{s} P_{R} b \bar{s} P_{R} b\right| \bar{B}_{s}\right\rangle & =-\frac{5}{24} m_{B_{s}} f_{B_{s}}^{2}\left(\frac{m_{B_{s}}}{m_{b}+m_{s}}\right)^{2},
\end{aligned}
$$

where $P_{L, R}=\left(1 \mp \gamma_{5}\right) / 2$ and $f_{B_{s}}=(238.8 \pm 9.5) \mathrm{MeV}$ [17]. An updated theoretical prediction can be found in Ref. [6], whose results we use in our numerical calculations.

Any NP contribution to $\Gamma_{12}^{s, \mathrm{NP}}$ must come from a new operator of the form $b \rightarrow$ $s f \bar{f}$, where $f$ is a light fermion. If $\Gamma_{12}^{s, \mathrm{NP}}$ is to be significant, it must be at least comparable to $\Gamma_{12}^{s, \mathrm{SM}}$. Now, most NP light fermionic operators are constrained to be small. In particular, (i) $e$ and $\mu$ loop contributions are strongly bounded by the $b \rightarrow s e^{+} e^{-}$and $b \rightarrow s \mu^{+} \mu^{-}$processes, (ii) the $b \rightarrow s u \bar{u}$ and $b \rightarrow s d \bar{d}$ operators are constrained by the measurement of $B(\bar{B} \rightarrow \pi K)$, (iii) $B(\bar{B} \rightarrow \phi K)$ constrains $b \rightarrow s s \bar{s}$. On the other hand, the bounds on the NP $b \rightarrow s \tau^{+} \tau^{-}$transition are very weak. For example, the present upper bound on $B\left(\bar{B}_{s} \rightarrow \tau^{+} \tau^{-}\right)$is only $\lesssim 5 \%$ [18], to be compared with the SM prediction of $B\left(\bar{B}_{s} \rightarrow \tau^{+} \tau^{-}\right) \sim 10^{-8}$. Also, the current upper bound on $B\left(\bar{B} \rightarrow X_{s} \tau^{+} \tau^{-}\right)$is just $\lesssim 5 \%$ [18]. For this reason, in the NP models we consider, we examine the contribution to $\Gamma_{12}^{s, \mathrm{NP}}$ coming from $b \rightarrow s \tau^{+} \tau^{-}$. 
Another potential important effect on $\Gamma_{12}^{s, \mathrm{NP}}$ comes from the NP $b \rightarrow s c \bar{c}$ operator. Although the constraints from $\bar{B} \rightarrow D D_{s}, \bar{B}_{d} \rightarrow J / \psi K_{S}$, etc. are such that the NP $c$-quark loop contribution cannot be large enough to compete with $\Gamma_{12}^{s, \mathrm{SM}}$, the SM-NP interference term can be significant.

The decay width difference $\Delta \Gamma_{s}$ has been measured independently. The angular analysis of $\bar{B}_{s} \rightarrow J / \psi \phi$ gives [8, 19, 20]

$$
\Delta \Gamma_{s}= \pm\left(0.154_{-0.070}^{+0.054}\right) \mathrm{ps}^{-1},
$$

to be compared with the the SM prediction [6]

$$
\Delta \Gamma_{s}^{\mathrm{SM}}=(0.096 \pm 0.039) \mathrm{ps}^{-1} .
$$

If NP contributes to $\Gamma_{12}^{s, \mathrm{NP}}$, it is present in the width difference, whose expression is given by

$$
\Delta \Gamma_{s}=\Delta \Gamma_{s}^{\mathrm{SM}}\left|\Xi_{s}\right| \cos \left(\phi_{M}^{s}-\phi_{\Gamma}^{s}\right) .
$$

The measurement of $\Delta \Gamma_{s}$ therefore constrains $\Gamma_{12}^{s, \mathrm{NP}}$.

There is another measurement which must be taken into account. The CDF 21] and $\mathrm{D} \varnothing$ [22] Collaborations have measured indirect CP violation in $\bar{B}_{s} \rightarrow J / \psi \phi$. They obtain $S_{\psi \phi}=-2 \beta_{s}$, and find [8]

$$
\beta_{s}=0.39_{-0.14}^{+0.18} \text { or } 1.18_{-0.18}^{+0.14} .
$$

This disagrees with the SM prediction

$$
\beta_{s}^{\mathrm{SM}}=0.019 \pm 0.001
$$

at $2 \sigma$. (Note: the recent CDF measurement agrees with the SM better than the previous DØand CDF measurements.) Now, we have assumed that the NP affects $M_{12}^{s}$, so that there is a weak phase in $B_{s}-\bar{B}_{s}$ mixing, $\phi_{M}^{s}$. Depending on which NP operator(s) contribute to $\Gamma_{12}^{s, \mathrm{NP}}$, there may also be a contribution to the decay of $\bar{B}_{s} \rightarrow J / \psi \phi$. Thus, the NP is constrained in that the $\mathrm{CDF} / \mathrm{D} \varnothing$ measurement must be reproduced.

To detail the contribution of the NP to the indirect CP asymmetry in $\bar{B}_{s} \rightarrow$ $J / \psi \phi$, we follow the procedure of Ref. [23]. First, we note that $\bar{B}_{s} \rightarrow J / \psi \phi$ is really three separate decays, one for each polarization state $\lambda$ of the final-state vector particles; longitudinal: $\lambda=0$, transverse: $\lambda=\{\|, \perp\}$. Second, in Ref. [24] it is argued that all strong phases associated with NP amplitudes are negligible. In this case, for each polarization one can combine all NP matrix elements into a single NP amplitude, with a single weak phase $\varphi_{\lambda}$ :

$$
\sum\left\langle(J / \psi \phi)_{\lambda}\left|\mathcal{O}_{\mathrm{NP}}\right| B_{s}\right\rangle=b_{\lambda} e^{i \varphi_{\lambda}} .
$$


We now assume that this single NP amplitude contributes to the decay of $\bar{B}_{s} \rightarrow$ $J / \psi \phi$. The decay amplitude for each of the three possible polarization states may then be written as

$$
\begin{aligned}
& A_{\lambda} \equiv A m p\left(B_{s} \rightarrow J / \psi \phi\right)_{\lambda}=a_{\lambda} e^{i\left(\delta_{\lambda}^{a}-\delta_{\perp}^{a}\right)}+b_{\lambda} e^{i \varphi_{\lambda}} e^{-i \delta_{\perp}^{a}}, \\
& \bar{A}_{\lambda} \equiv A m p\left(\bar{B}_{s} \rightarrow J / \psi \phi\right)_{\lambda}=a_{\lambda} e^{i\left(\delta_{\lambda}^{a}-\delta_{\perp}^{a}\right)}+b_{\lambda} e^{-i \varphi_{\lambda}} e^{-i \delta_{\perp}^{a}},
\end{aligned}
$$

where $a_{\lambda}$ and $b_{\lambda}$ represent the SM and NP amplitudes, respectively, $\varphi_{\lambda}$ is the newphysics weak phase, and the $\delta_{\lambda}^{a}$ are the SM strong phases. All strong phases are given relative to $\delta_{\perp}^{a} . a_{\lambda}$ is defined to be positive for every polarization. $b_{\lambda}$ can also be taken to be positive: if it is negative, the minus sign can be absorbed in the weak phase by redefining $\varphi_{\lambda} \rightarrow \varphi_{\lambda}+\pi$. We emphasize this fact by writing the ratio $b_{\lambda} / a_{\lambda}$ as the positive-definite quantity $\left|r_{\lambda}\right|$. Note that strong phases are generated by rescattering, and this costs a factor of about 25. The strong phase of the SM color-suppressed $\bar{b} \rightarrow \bar{c} c \bar{s}$ diagram $C$ is generated by rescattering of the color-allowed $\bar{b} \rightarrow \bar{c} c \bar{s}$ tree diagram $T$. Since $|C / T|$ is expected to be in the range 0.2-0.6, the SM strong phase is on the small side, but is not negligible.

Putting all this together, we find that the indirect CP asymmetry in $\bar{B}_{s} \rightarrow J / \psi \phi$ measures

$$
S_{\psi \phi}=\sin \phi_{M}^{s}+2\left|r_{\lambda}\right| \cos \phi_{M}^{s} \sin \varphi_{\lambda} \cos \delta_{\lambda}^{a} .
$$

(We have neglected the SM contribution to the weak phase of $B_{s}-\bar{B}_{s}$ mixing, which is expected to be only $\sim 2 \%$ [Eq. (2.7)].) If the NP contribution to $\Gamma_{12}^{s, \mathrm{NP}}$ comes only from $b \rightarrow s \tau^{+} \tau^{-}$, then $r_{\lambda}=0$ and $S_{\psi \phi}=\sin \phi_{M}^{s}$. However, if the NP $b \rightarrow s c \bar{c}$ operator is involved in $\Gamma_{12}^{s, \mathrm{NP}}$, then $r_{\lambda} \neq 0$ and the full expression for $S_{\psi \phi}$ above must be used.

Finally, we note that the phases of $a_{\mathrm{sl}}^{s}$ and $S_{\psi \phi}$ are different when there is a NP contribution to $\Gamma_{12}^{s}$ and/or $\bar{B}_{s} \rightarrow J / \psi \phi$. In addition, the relation between them [25],

$$
a_{\mathrm{sl}}^{s}=-\frac{\left|\Delta \Gamma_{s}\right|}{\left|\Delta M_{s}\right|} S_{\psi \phi} / \sqrt{1-S_{\psi \phi}^{2}},
$$

is violated in this case.

\section{New-Physics Models}

\subsection{Z-mediated FCNC's}

In the model with $Z$-mediated FCNC's ( $Z$ FCNC), a new vector-like isosinglet downtype quark $d^{\prime}$ is added to the particle spectrum [26, 27, 28. Such quarks appear in $E_{6}$ GUT theories, for example. The ordinary $Q_{e m}=-1 / 3$ quarks mix with the $d^{\prime}$. Because the $d_{L}^{\prime}$ has a different $I_{3 L}$ from $d_{L}, s_{L}$ and $b_{L}$, FCNC's appear at tree level in the left-handed sector. In particular, a $Z \bar{b} s$ coupling can be generated:

$$
\mathcal{L}_{F C N C}^{Z}=-\frac{g}{2 \cos \theta_{W}} U_{s b} \bar{s} \gamma^{\mu} P_{L} b Z_{\mu}+\text { h.c. }
$$


This coupling leads to a NP contribution to $B_{s}-\bar{B}_{s}$ mixing at tree level.

The SM contribution to $M_{12}^{s}$ is

$$
M_{12}^{s, \mathrm{SM}}=\frac{G_{F}^{2}}{12 \pi^{2}} M_{W}^{2} \lambda_{t}^{2} \eta_{B} B_{B_{s}} f_{B_{s}}^{2} M_{B_{s}} E\left(x_{t}\right),
$$

where $\lambda_{t}=V_{t b} V_{t s}^{*}, x_{t}=m_{t}^{2} / M_{W}^{2}, \eta_{B} \simeq 0.551$ is the QCD correction, and we take the hadronic parameter $f_{B_{s}} B_{B_{s}}^{1 / 2}=295 \pm 36 \mathrm{MeV}$. The loop function $E\left(x_{t}\right)$ is given by

$$
E\left(x_{t}\right)=\frac{-4 x_{t}+11 x_{t}^{2}-x_{t}^{3}}{4\left(1-x_{t}\right)^{2}}+\frac{3 x_{t}^{3} \ln x_{t}}{2\left(1-x_{t}\right)^{3}} .
$$

The mass difference $\Delta M_{s}$ in the $Z \mathrm{FCNC}$ model is given by [28]

$$
\Delta M_{s}=\Delta M_{s}^{\mathrm{SM}}\left|1+a\left(\frac{U_{s b}}{\lambda_{t}}\right)-b\left(\frac{U_{s b}}{\lambda_{t}}\right)^{2}\right|,
$$

where

$$
a=4 \frac{C\left(x_{t}\right)}{E\left(x_{t}\right)}, \quad b=\frac{2 \sqrt{2} \pi^{2}}{G_{F} M_{W}^{2} E\left(x_{t}\right)} .
$$

The loop functions $E\left(x_{t}\right)$ and $C\left(x_{t}\right)$ are given by [28]

$$
\begin{aligned}
& E\left(x_{t}\right)=\frac{-4 x_{t}+11 x_{t}^{2}-x_{t}^{3}}{4\left(1-x_{t}\right)^{2}}+\frac{3 x_{t}^{3} \ln x_{t}}{2\left(1-x_{t}\right)^{3}}, \\
& C\left(x_{t}\right)=\frac{x_{t}}{4}\left[\frac{4-x_{t}}{1-x_{t}}+\frac{3 x_{t} \ln x_{t}}{\left(1-x_{t}\right)^{2}}\right] .
\end{aligned}
$$

The term in Eq. (3.4) proportional to $a$ is obtained from a diagram with both SM and NP $Z$ vertices; that proportional to $b$ corresponds to the diagram with two NP $Z$ vertices.

This coupling will also lead to contributions to $\Gamma_{12}^{s}$ due to the generation of the $Z$-mediated operator $b \rightarrow s f \bar{f}(f=\tau, c)$. The amplitude is

$$
\frac{G_{F}}{\sqrt{2}} U_{s b} \bar{s} \gamma_{\mu}\left(1-\gamma_{5}\right) b \bar{f} \gamma^{\mu}\left(I_{3}-Q \sin ^{2} \theta_{W}\right)\left(1 \mp \gamma_{5}\right) f .
$$

There are diagrams with two NP vertices, and with one SM and one NP vertex. The dominant contribution is due to SM-NP interference, yielding

$$
\begin{gathered}
\Gamma_{12}^{s, Z}=-\frac{G_{F}^{2} m_{b}^{2} \lambda_{c} U_{s b}}{\pi} \sqrt{1-4 x_{c}} \times\left[\left\{\frac{1}{2}\left(1-x_{c}\right)-\frac{2}{3} \sin ^{2} \theta_{W}\left(1+2 x_{c}\right)\right\}\left\langle O_{L L}\right\rangle\right. \\
\left.+\left(\frac{1}{2}-\frac{2}{3} \sin ^{2} \theta_{W}\right)\left(1+2 x_{c}\right)\left\langle\widetilde{O}_{R R}\right\rangle\right] .
\end{gathered}
$$




\section{$3.2 Z^{\prime}$-mediated FCNC's}

In the model with $Z^{\prime}$-mediated FCNC's $\left(Z^{\prime} \mathrm{FCNC}\right)$, the gauge group contains an additional $U(1)^{\prime}$, which leads to a $Z^{\prime}$ [29]. Within some string-construction or GUT models such as $E_{6}$, it is possible to have family non-universal $Z^{\prime}$ couplings. In the physical basis, FCNC's generally appear at tree level in both the left-handed (LH) and right-handed $(\mathrm{RH})$ sectors. In particular, the interaction Lagrangian can contain $Z^{\prime} \bar{b} s$ couplings:

$$
\mathcal{L}_{F C N C}^{Z^{\prime}}=-\frac{g}{\cos \theta_{W}}\left[\bar{s} \gamma^{\mu} P_{L} B_{s b}^{L} b+(L \leftrightarrow R)\right] Z_{\mu}^{\prime}+\text { h.c. },
$$

which lead to a tree-level contribution to $B_{s}-\bar{B}_{s}$ mixing. (Note: the $U(1)^{\prime}$ coupling constant is $g^{\prime}$. However, the above Lagrangian is written in terms of the SM coupling; the ratio of the two couplings is absorbed into the $B_{s b}$ 's. This makes the comparison of NP and SM effects more straightforward.)

The effective Hamiltonian for $M_{12}^{s}$ in the $Z^{\prime} \mathrm{FCNC}$ model at the scale $m_{b}$ is calculated from the tree-level diagrams [30, 31]:

$$
\begin{aligned}
\mathcal{H}_{\mathrm{eff}}=\frac{4 G_{F}}{\sqrt{2}} \frac{m_{Z}^{2}}{m_{Z^{\prime}}^{2}}[ & \eta^{6 / 23}\left(B_{s b}^{L}\right)^{2} O_{L L}\left(m_{b}\right)+\eta^{6 / 23}\left(B_{s b}^{R}\right)^{2} O_{R R}\left(m_{b}\right)+2 \eta^{3 / 23} B_{s b}^{L} B_{s b}^{R} O_{L R}\left(m_{b}\right) \\
& \left.+\frac{4}{3}\left(\eta^{3 / 23}-\eta^{-24 / 23}\right) B_{s b}^{L} B_{s b}^{R} \widetilde{O}_{L R}\left(m_{b}\right)\right]
\end{aligned}
$$

where $\eta=\alpha_{s}\left(M_{Z^{\prime}}\right) / \alpha_{s}\left(m_{b}\right)$ and

$$
\begin{aligned}
O_{L L} & =\bar{s} \gamma_{\mu} P_{L} b \bar{s} \gamma^{\mu} P_{L} b, \\
O_{R R} & =\bar{s} \gamma_{\mu} P_{R} b \bar{s} \gamma^{\mu} P_{R} b, \\
O_{L R} & =\bar{s} \gamma_{\mu} P_{L} b \bar{s} \gamma^{\mu} P_{R} b, \\
\widetilde{O}_{L R} & =\bar{s} P_{L} b \bar{s} P_{R} b .
\end{aligned}
$$

In addition to the SM operator $O_{L L}$, the operators $O_{R R}$ and $O_{L R}$ are generated at the scale $M_{Z^{\prime}}$. The operator $\widetilde{O}_{L R}$ is generated through renormalization down to the scale $m_{b}$.

The matrix element $M_{12}^{s, Z^{\prime}}$ for $B_{s}-\bar{B}_{s}$ mixing is given by

$$
M_{12}^{s, Z^{\prime}}=\left\langle B_{s}\left|\mathcal{H}_{\mathrm{eff}}\right| \bar{B}_{s}\right\rangle .
$$

The hadronic matrix elements are again calculated using the vacuum insertion approximation [16]. $\left\langle B_{s}\left|O_{L L}\right| \bar{B}_{s}\right\rangle$ is given in Eq. (2.2), $\left\langle O_{R R}\right\rangle \equiv\left\langle B_{s}\left|O_{R R}\right| \bar{B}_{s}\right\rangle=$ $\left\langle B_{s}\left|O_{L L}\right| \bar{B}_{s}\right\rangle$, and

$$
\begin{aligned}
& \left\langle O_{L R}\right\rangle \equiv\left\langle B_{s}\left|O_{L R}\right| \bar{B}_{s}\right\rangle=-\left[\frac{1}{4}+\frac{1}{6}\left(\frac{m_{B_{s}}}{m_{b}+m_{s}}\right)^{2}\right] m_{B_{s}} f_{B_{s}}^{2}, \\
& \left\langle\widetilde{O}_{L R}\right\rangle \equiv\left\langle B_{s}\left|\widetilde{O}_{L R}\right| \bar{B}_{s}\right\rangle=\left[\frac{1}{24}+\frac{1}{4}\left(\frac{m_{B_{s}}}{m_{b}+m_{s}}\right)^{2}\right] m_{B_{s}} f_{B_{s}}^{2} .
\end{aligned}
$$


The $Z^{\prime}$ contribution to $\Gamma_{12}^{s}$ through $\tau$ pairs is obtained from the formulae given in Refs. [32, 33]:

$$
\begin{aligned}
\Gamma_{12}^{s, Z^{\prime}}= & -\frac{4 G_{F}^{2} m_{b}^{2}}{3 \pi}\left(\frac{m_{Z}^{2}}{m_{Z^{\prime}}^{2}}\right)^{2} \sqrt{1-4 x_{\tau}} \times \\
& {\left[\left\{\left(B^{L L}\right)^{2}+\left(B^{R R}\right)^{2}+\left(B^{L R}\right)^{2}+\left(B^{R L}\right)^{2}\right\}\left\{\left(1-x_{\tau}\right)\left\langle O_{L L}\right\rangle+\left(1+2 x_{\tau}\right)\left\langle\widetilde{O}_{R R}\right\rangle\right\}\right.} \\
& +2\left\{B^{L L} B^{R L}+B^{R R} B^{L R}\right\}\left\{\left(1-x_{\tau}\right)\left\langle O_{L R}\right\rangle+\left(1+2 x_{\tau}\right)\left\langle\widetilde{O}_{L R}\right\rangle\right\} \\
& +6\left\{B^{L L} B^{L R}+B^{R R} B^{R L}\right\} x_{\tau}\left\langle O_{L L}\right\rangle \\
& \left.+6\left\{B^{L L} B^{R R}+B^{L R} B^{R L}\right\} x_{\tau}\left\langle O_{L R}\right\rangle\right],
\end{aligned}
$$

where $B^{i j}=B_{s b}^{i} B_{\tau \tau}^{j}(i, j=L, R), x_{\tau}=m_{\tau}^{2} / m_{b}^{2}$. Comparing Eqs. (2.1) and (3.14), we can see that if $B^{i j} \sim\left(\lambda_{c} m_{Z^{\prime}}^{2} / m_{Z}^{2}\right)^{2} \sim \mathcal{O}(1), \Gamma_{12}^{s, Z^{\prime}}$ can be comparable to $\Gamma_{12}^{s, \mathrm{SM}}$. We will see that these $\mathcal{O}(1)$ couplings are still allowed by $B\left(\bar{B}_{s} \rightarrow \tau^{+} \tau^{-}\right)$and $B(\bar{B} \rightarrow$ $\left.X_{s} \tau^{+} \tau^{-}\right)$.

Although the $c$-quark loop contribution with both couplings from NP cannot be large enough to compete with the SM contribution, there can be an interference term between the SM and the NP in this case. This term is given by

$$
\begin{aligned}
& \Gamma_{12}^{s, Z^{\prime}+\mathrm{SM}}=-\frac{G_{F}^{2} m_{b}^{2} \lambda_{c}}{\pi}\left(\frac{m_{Z}^{2}}{m_{Z^{\prime}}^{2}}\right) \sqrt{1-4 x_{c}} \\
\times & {\left[4 B_{s b}^{L} B_{c c}^{L}\left\{\left(1-x_{c}\right)\left\langle O_{L L}\right\rangle+\left(1+2 x_{c}\right)\left\langle\widetilde{O}_{L R}\right\rangle\right\}+6 B_{s b}^{L} B_{c c}^{R} x_{c}\left\langle O_{L L}\right\rangle+12 B_{s b}^{R} B_{c c}^{R}\left\langle O_{L R}\right\rangle\right.} \\
+ & \left.4 B_{s b}^{R} B_{c c}^{L}\left\{\left(1-x_{c}\right)\left\langle O_{L R}\right\rangle+\left(1+2 x_{c}\right)\left\langle\widetilde{O}_{L R}\right\rangle\right\}\right] .
\end{aligned}
$$

Here again Eq. (3.15) can be comparable with $\Gamma_{12}^{s, \mathrm{SM}}$ when $B_{s b}^{i} B_{c c}^{j} \sim \mathcal{O}(1)(i, j=$ $L, R)$.

\section{Numerical Results}

\subsection{ZFCNC}

In the numerical study of the $Z \mathrm{FCNC}$ model, we scan the allowed values of the couplings after imposing the constraints from $\left|\Delta_{s}\right| \equiv \Delta M_{s} / \Delta M_{s}^{\mathrm{SM}}[$ [Eq. (3.4)] and $B\left(\bar{B} \rightarrow X_{s} \mu^{+} \mu^{-}\right)^{1}$. The general expression for the branching ratio of $\bar{B} \rightarrow X_{s} l^{+} l^{-}$,

\footnotetext{
${ }^{1}$ There can be other constraints, such as those from the forward-backward asymmetry in $B \rightarrow$ $K^{*} l^{+} l^{-}$35. However, we do not include these in our analysis, since, as can be seen below in Fig. 2, the two constraints used are sufficient to rule out the ZFCNC model as an explanation of $S_{\psi \phi}$ and $a_{\mathrm{sl}}^{s}$.
} 
where $l=e, \mu, \tau$, is given by 34

$$
B\left(\bar{B} \rightarrow X_{s} l^{+} l^{-}\right)=B_{0} \int_{4 m_{l}^{2} / m_{b}^{2}}^{\left(1-m_{s} / m_{b}\right)^{2}}\left(B_{S M}(z)+B_{S M-V A}(z)+B_{V A}(z)\right) d z .
$$

The expressions for $B_{0}, B_{S M}, B_{S M-V A}$, and $B_{V A}$ are given in Ref. [34. We do not repeat them here. We note only that the integrand depends on the NP couplings $R_{V}, R_{A}, R_{V}^{\prime}$ and $R_{A}^{\prime}$. In the $Z \mathrm{FCNC}$ model, the NP couplings $R_{V, A}^{\prime}=0$, and the $R_{V, A}$ are given by 36]

$$
R_{V}=\frac{2 \pi}{\alpha} \frac{U_{s b}}{V_{t b} V_{t s}^{*}}\left(-\frac{1}{2}+2 \sin ^{2} \theta_{W}\right), \quad R_{A}=\frac{\pi}{\alpha} \frac{U_{s b}}{V_{t b} V_{t s}^{*}} .
$$

We define $q^{\mu}$ as the sum of the 4-momenta of the $\mu^{+}$and $\mu^{-}$in $\bar{B} \rightarrow X_{s} \mu^{+} \mu^{-}$. The theoretical predictions for the branching ratio of this decay are not reliable over the whole $q^{2}$ region due to the presence of charm resonances at intermediate $q^{2}\left(7 \mathrm{GeV}^{2} \leq q^{2} \leq 12 \mathrm{GeV}^{2}\right)$. The predictions are relatively more robust for low $q^{2}\left(1 \mathrm{GeV}^{2} \leq q^{2} \leq 6 \mathrm{GeV}^{2}\right)$ and high $q^{2}\left(14.4 \mathrm{GeV}^{2} \leq q^{2} \leq m_{b}^{2}\right)$. However, the two regions have different sensitivities to the short-distance physics. The dominant contribution to $\bar{B} \rightarrow X_{s} \mu^{+} \mu^{-}$in the low- $q^{2}$ region comes from a virtual photon, whereas the dominant contribution in the high- $q^{2}$ region comes from the $Z$ and $W$. Since we are interested specifically in the $Z \bar{b} s$ coupling, we use the branching ratio in the high- $q^{2}$ region to constrain the $Z$ FCNC parameter space.

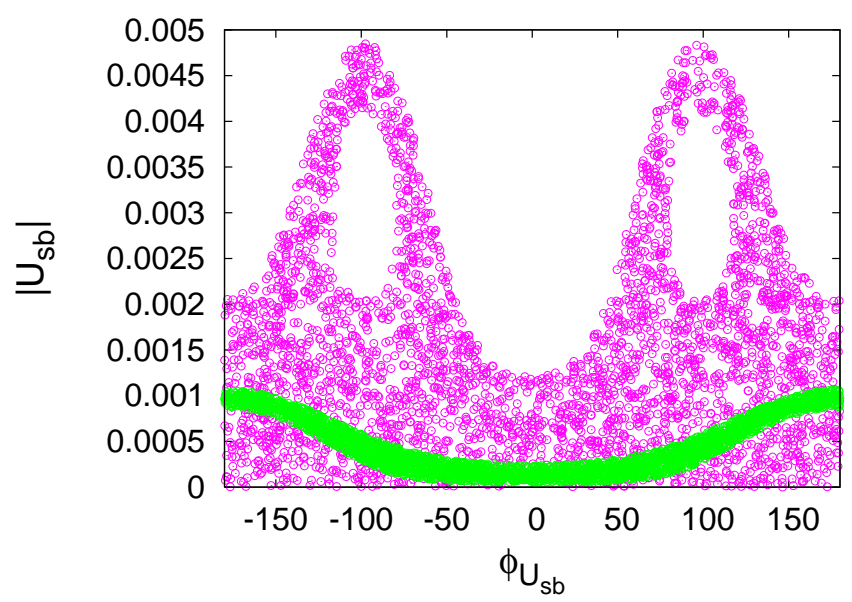

Figure 1: The $\phi_{U_{s b}}\left|U_{s b}\right|$ parameter space allowed by $\left|\Delta_{s}\right|$ (magenta) and $B(\bar{B} \rightarrow$ $X_{s} \mu^{+} \mu^{-}$) (green).

As described earlier, $U_{s b}$ denotes the $Z \bar{b} s$ coupling generated in the $Z$ FCNC model [Eq. (3.1)]. The parameters of the model are therefore the magnitude and the phase of this coupling, $\left|U_{s b}\right|$ and $\phi_{U_{s b}} \equiv \arg U_{s b}$. Fig. 1 shows the allowed region in $\left(\phi_{U_{s b}},\left|U_{s b}\right|\right)$ space due to the measurements of $\left|\Delta_{s}\right|$ (magenta) and $B\left(\bar{B} \rightarrow X_{s} \mu^{+} \mu^{-}\right)$ 
(green). Using constraints only from $\left|\Delta_{s}\right|$, we see that the full range of $\phi_{U_{s b}}$ is obtained only when $\left|U_{s b}\right| \lesssim 0.001$, while $\left|U_{s b}\right|$ can be as large as 0.005 in the constrained regions of $\phi_{U_{s b}}$. However, if we include the constraints from $B\left(\bar{B} \rightarrow X_{s} \mu^{+} \mu^{-}\right)$, the $\left(\phi_{U_{s b}}\right.$, $\left.\left|U_{s b}\right|\right)$ space is reduced considerably. From Fig. 1, one sees that $B\left(\bar{B} \rightarrow X_{s} \mu^{+} \mu^{-}\right)$ limits the upper value of $\left|U_{s b}\right|$ to be around 0.001 .

Using these allowed couplings, we can calculate the observables $S_{\psi \phi}, a_{\mathrm{sl}}^{s}$ and $\Delta \Gamma_{s}$, and compare these values with the measurements. The $1 \sigma$ ranges of these observables are

$$
\begin{aligned}
S_{\psi \phi} & :[-0.91,-0.47], \\
a_{\mathrm{sl}}^{s} & :[-17.7,-7.7] \times 10^{-3}, \\
\Delta \Gamma_{s} & : \pm[0.084,0.208] \mathrm{ps}^{-1} .
\end{aligned}
$$

In addition, we can examine correlations between the observables.
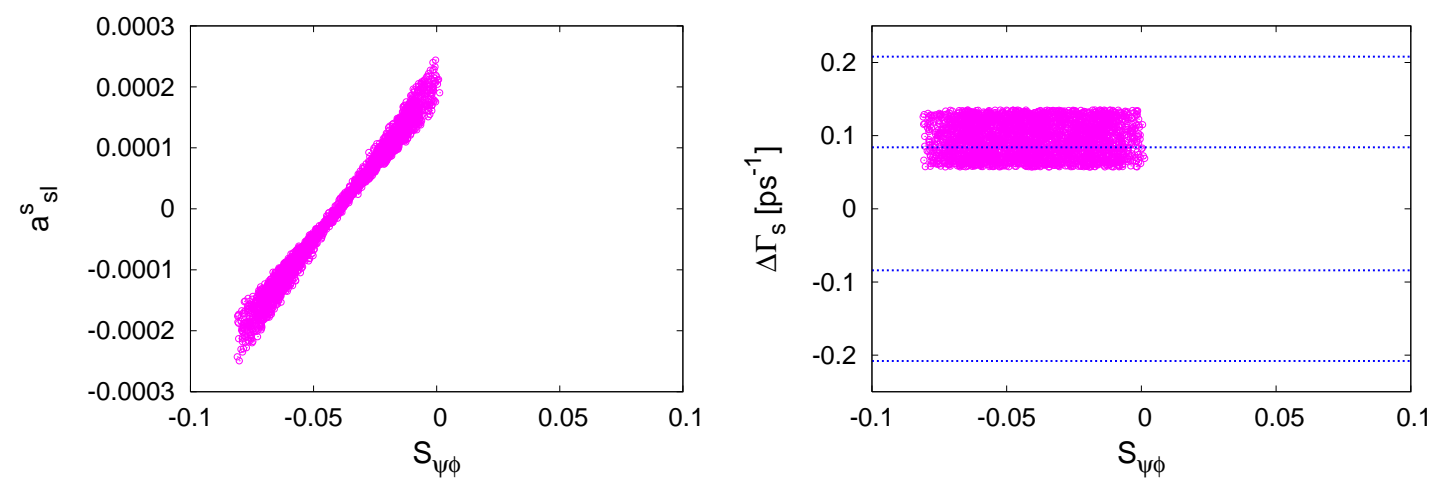

Figure 2: The $a_{s l^{-}}^{s} S_{\psi \phi}$ correlation plot (left panel) and the $\Delta \Gamma_{s^{-}} S_{\psi_{\phi}}$ correlation plot (right panel). The horizontal lines in the right panel indicate the $1 \sigma$ experimental allowed range for $\Delta \Gamma_{s}$.

As a consequence of the strong constraint on the size and phase of $U_{s b}$, we see in Fig. 2 that it is not possible to explain the present $1 \sigma$ ranges of $S_{\psi \phi}$ and $a_{\mathrm{sl}}^{s}$ in the $Z$ FCNC model. Still, this model can produce values for these observables which are closer to the experimental data. In particular, one can have $a_{\mathrm{sl}}^{s} \sim-3 \times 10^{-4}$, which is about 10 times larger in magnitude than the SM prediction of $\sim 2 \times 10^{-5}$. And $S_{\psi \phi}$ can be decreased to -0.08 , as compared to the SM value of -0.04 .

\section{2 $Z^{\prime} \mathrm{FCNC}$}

As discussed earlier, a large NP contribution to $\Gamma_{12}^{s, \mathrm{NP}}$, and hence to $a_{s l}^{s}$, may come from the NP $b \rightarrow s \tau^{+} \tau^{-}$and/or $b \rightarrow s c \bar{c}$ operators. Since the two operators need not be related to one another, a large NP effect on $b \rightarrow s \tau^{+} \tau^{-}$but not on $b \rightarrow s c \bar{c}$, or vice versa, is conceivable. Thus, in analyzing the $Z^{\prime} \mathrm{FCNC}$ model, we examine 
whether it is possible to explain the present $1 \sigma$ measurement of $a_{s l}^{s}$ if only one of the NP operators is present.

\subsubsection{NP $b \rightarrow s \tau^{+} \tau^{-}$operator only}

We first consider the case where the NP contribution to $\Gamma_{12}^{s, \mathrm{NP}}$ comes only from the $b \rightarrow s \tau^{+} \tau^{-}$operator. In our numerical study we scan the allowed values of the $Z^{\prime} \mathrm{FCNC}$ couplings imposing the constraints from $\left|\Delta_{s}\right|, B\left(\bar{B} \rightarrow X_{s} \tau^{+} \tau^{-}\right)<5 \%$ and $B\left(\bar{B}_{s} \rightarrow \tau^{+} \tau^{-}\right)<5 \%$. $\left|\Delta_{s}\right|$ can be obtained using Eqs. (3.10) and (3.12), and $B\left(\bar{B} \rightarrow X_{s} \tau^{+} \tau^{-}\right)$is given by Eq. (4.1) with $l=\tau$. The branching ratio of $\bar{B}_{s} \rightarrow \tau^{+} \tau^{-}$is 34

$$
B\left(\bar{B}_{s} \rightarrow \tau^{+} \tau^{-}\right)=\frac{G_{F}^{2} \alpha^{2} m_{B_{s}}^{5} f_{B_{s}}^{2} \tau_{B_{s}}}{64 \pi^{3}}\left|V_{t b} V_{t s}^{*}\right|^{2} \sqrt{1-\frac{4 m_{\tau}^{2}}{m_{B_{s}}^{2}}}\left|\frac{2 m_{\tau}}{m_{B_{s}}^{2}}\left(C_{10}+R_{A}-R_{A}^{\prime}\right)\right|^{2} .
$$

In the $Z^{\prime} \mathrm{FCNC}$ model, the NP couplings are given by

$$
\begin{aligned}
& R_{V}=-\frac{4 \pi}{\alpha} \frac{m_{Z}^{2} / m_{Z^{\prime}}^{2}}{V_{t b} V_{t s}^{*}} B_{s b}^{L}\left(B_{\tau \tau}^{L}+B_{\tau \tau}^{R}\right) \\
& R_{A}=\frac{4 \pi}{\alpha} \frac{m_{Z}^{2} / m_{Z^{\prime}}^{2}}{V_{t b} V_{t s}^{*}} B_{s b}^{L}\left(B_{\tau \tau}^{L}-B_{\tau \tau}^{R}\right) \\
& R_{V}^{\prime}=-\frac{4 \pi}{\alpha} \frac{m_{Z}^{2} / m_{Z^{\prime}}^{2}}{V_{t b} V_{t s}^{*}} B_{s b}^{R}\left(B_{\tau \tau}^{L}+B_{\tau \tau}^{R}\right) \\
& R_{A}^{\prime}=\frac{4 \pi}{\alpha} \frac{m_{Z}^{2} / m_{Z^{\prime}}^{2}}{V_{t b} V_{t s}^{*}} B_{s b}^{R}\left(B_{\tau \tau}^{L}-B_{\tau \tau}^{R}\right)
\end{aligned}
$$

These couplings are constrained by the above observables. However, the key point is that, because the bounds on the NP $b \rightarrow s \tau^{+} \tau^{-}$transition are still weak, the constraints are not severe.

Fig. 3 shows the $a_{s l}^{s}-S_{\psi \phi}$ and $\Delta \Gamma_{s^{-}} S_{\psi \phi}$ correlation plots for the case where only $B_{s b}^{L}$ and $B_{\tau \tau}^{L, R}$ couplings are present. We make the following observations:

- $S_{\psi \phi}$ can take any value between -1 and 1 .

- The $Z^{\prime} \mathrm{FCNC}$ model gives values for $a_{s l}^{s}$ within its $1 \sigma$ range.

- One can simultaneously reproduce the present measurements of all three observables, $S_{\psi \phi}, a_{s l}^{s}$ and $\Delta \Gamma_{s}$, within their $1 \sigma$ ranges.

Obviously, one obtains the same conclusions if a nonzero $B_{s b}^{R}$ is also present.

\subsubsection{NP $b \rightarrow s c \bar{c}$ operator only}

We now consider the case where the NP contribution to $\Gamma_{12}^{s, \mathrm{NP}}$ comes only from the $b \rightarrow s c \bar{c}$ operator. In our numerical study we scan the allowed values of the $Z^{\prime} \mathrm{FCNC}$ 

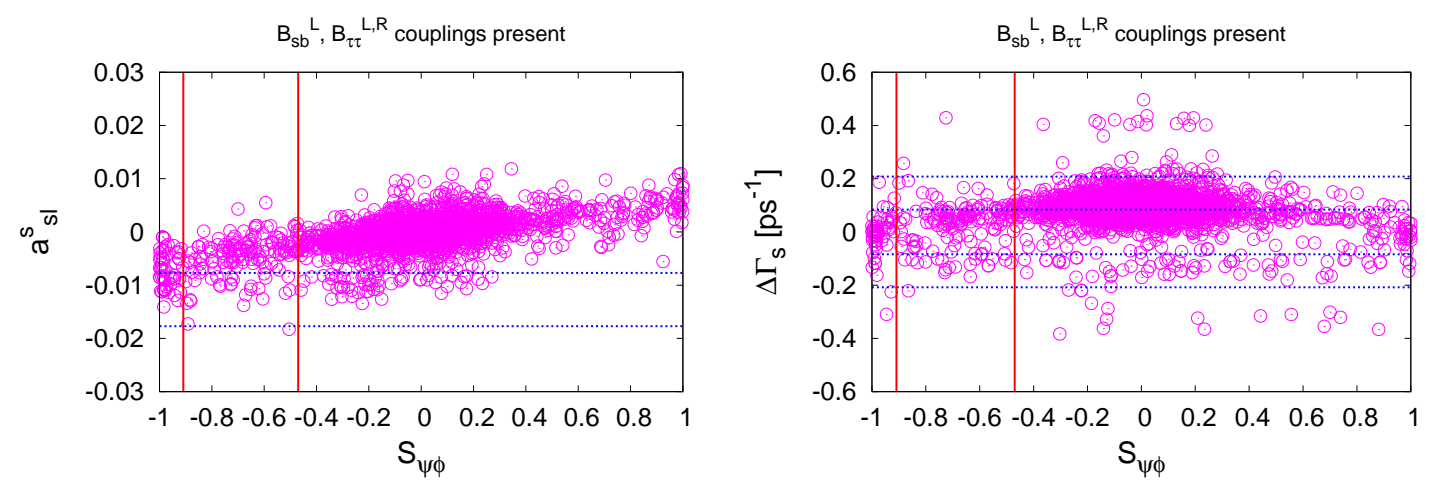

Figure 3: The $a_{s l^{-}}^{s} S_{\psi \phi}$ correlation plot (left panel) and the $\Delta \Gamma_{s^{-}} S_{\psi \phi}$ correlation plot (right panel), for the case where only $B_{s b}^{L}$ and $B_{\tau \tau}^{L, R}$ couplings are present. The horizontal and vertical lines indicate the $1 \sigma$ experimental allowed ranges for the observables.

couplings imposing the constraints from $\left|\Delta_{s}\right|$ and the indirect CP asymmetry in $\bar{B}_{d} \rightarrow J / \psi K_{S}$. This latter constraint arises as follows. In the presence of NP in the decay $b \rightarrow s c \bar{c}$, the effective measured $\sin 2 \beta$ in $\bar{B}_{d} \rightarrow J / \psi K_{S}$ is given by [23]

$$
\sin 2 \beta^{\text {meas }}=\sin 2 \beta+2|r| \cos 2 \beta \sin \varphi \cos \delta^{a} .
$$

Here, the NP parameters are defined similarly to those in Eq. (2.10): $|r|$ is the ratio of magnitudes of the NP and SM $b \rightarrow s c \bar{c}$ amplitudes, $\varphi$ is the NP weak phase, and $\delta^{a}$ is the SM strong phase. The true value of $\sin 2 \beta$ is taken from the fit to the sides of the unitarity triangle: $\sin 2 \beta=0.731 \pm 0.038$, while the experimental measurement gives $\sin 2 \beta^{\text {meas }}=0.668 \pm 0.028$ [37]. Using these values, $|r|$ is estimated to be [23]

$$
|r|=(4.6 \pm 3.5) \% \text {. }
$$

Thus, at $1 \sigma,|r| \leq 8.1 \%$ is permitted. This upper bound on $|r|$, along with the measurement of $\left|\Delta_{s}\right|$, constrain the NP couplings.

Fig. t shows the $a_{s l}^{s}-S_{\psi \phi}$ and $\Delta \Gamma_{s}-S_{\psi \phi}$ correlation plots for the case where only $B_{s b}^{L}$ and $B_{c c}^{L, R}$ couplings are present. The results are the same as for the case where a NP $b \rightarrow s \tau^{+} \tau^{-}$operator was added:

- $S_{\psi \phi}$ can take any value between -1 and 1 .

- The $Z^{\prime} \mathrm{FCNC}$ model gives values for $a_{s l}^{s}$ within its $1 \sigma$ range.

- One can simultaneously reproduce the present measurements of all three observables, $S_{\psi \phi}, a_{s l}^{s}$ and $\Delta \Gamma_{s}$, within their $1 \sigma$ ranges.

We therefore see that the $Z^{\prime} \mathrm{FCNC}$ model can reproduce the present measurements of $S_{\psi \phi}, \Delta \Gamma_{s}$ and $a_{\mathrm{sl}}^{s}$ within their $1 \sigma$ ranges if the NP contribution comes only from either the $b \rightarrow s \tau^{+} \tau^{-}$or $b \rightarrow s c \bar{c}$ operators. 

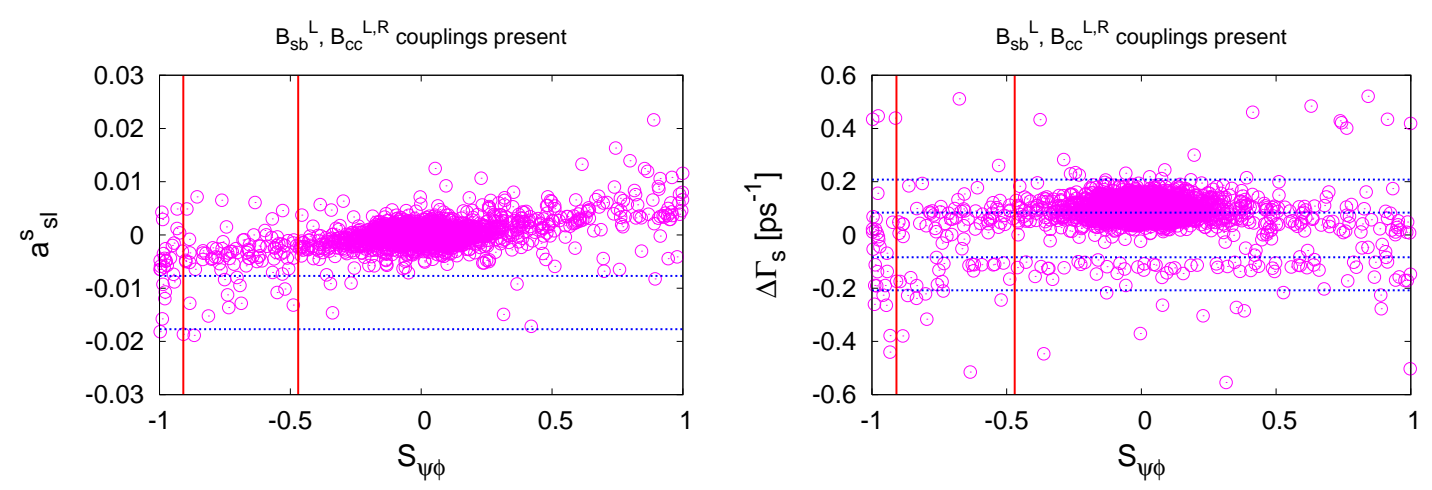

Figure 4: The $a_{s l}^{s}-S_{\psi \phi}$ correlation plot (left panel) and the $\Delta \Gamma_{s^{-}} S_{\psi \phi}$ correlation plot (right panel), for the case where only $B_{s b}^{L}$ and $B_{c c}^{L, R}$ couplings are present. The horizontal and vertical lines indicate the $1 \sigma$ experimental allowed ranges for the observables.

\section{Conclusions}

Recently, the $\mathrm{D} \varnothing$ Collaboration measured the CP-violating like-sign dimuon charge asymmetry in neutral $B$ decays and found a $3.2 \sigma$ difference from the prediction of the standard model (SM). Combining the DØ result with other measurements, we obtain the semileptonic charge asymmetry for $B_{s}$ mesons, $\left(a_{\mathrm{sl}}^{s}\right)_{\text {ave }}=-(12.7 \pm 5.0) \times 10^{-3}$, which is still $\sim 2.5 \sigma$ away from the $\mathrm{SM}$ prediction.

A non-SM $a_{\mathrm{sl}}^{s}$ can be explained theoretically by a new-physics (NP) contribution to $B_{s}-\bar{B}_{s}$ mixing, specifically to $M_{12}^{s}$ and $\Gamma_{12}^{s}$, the dispersive and absorptive parts of the mixing. It is usually assumed that $\Gamma_{12}^{s}$ is dominated by the SM tree-level $b \rightarrow s c \bar{c}$ coupling, and that it is not affected by the NP. Instead, NP is considered only in $M_{12}^{s}$. However, in this case, the measured value of $\left(a_{\mathrm{sl}}^{s}\right)$ ave within its $1 \sigma$ range cannot be explained. Thus, NP in $\Gamma_{12}^{s}$ is necessary.

There are some NP models (leptoquarks, R-parity-violating SUSY) whose contributions to $\Gamma_{12}^{s}$ can compete with the SM. In this paper, we examine models with flavor-changing neutral gauge bosons $\left(Z\right.$ or $\left.Z^{\prime}\right)$ to see if they can explain $\left(a_{\mathrm{sl}}^{s}\right)_{\text {ave }}$. The models must also reproduce the measured values of the indirect CP asymmetry $S_{\psi \phi}$ in $\bar{B}_{s} \rightarrow J / \psi \phi$ and $\Delta \Gamma_{s}$.

In our models we assume that the main contribution to $\Gamma_{12}^{s}$ comes from diagrams involving $\tau$ and/or $c$ loops. The point is that the current constraints on NP $b \rightarrow s f \bar{f}$ $(f=\tau, c)$ transitions are quite weak. We find that the model with flavor-changing $Z$ couplings $(Z \mathrm{FCNC})$ cannot reproduce the present measured values of $S_{\psi \phi}$ and $a_{\mathrm{sl}}^{s}$ within their $1 \sigma$ ranges. Still, the $Z$ FCNC model can lead to modified values for $S_{\psi \phi}$ and $a_{\mathrm{sl}}^{s}$ which are closer to the experimental data than the SM predictions.

On the other hand, in the model with flavor-changing $Z^{\prime}$ couplings ( $Z^{\prime} \mathrm{FCNC}$ ), even after imposing the current experimental constraints, the present measurements of $S_{\psi \phi}, a_{s l}^{s}$ and $\Delta \Gamma_{s}$, can all be simultaneously reproduced within their $1 \sigma$ ranges. 
Indeed, the full range of $S_{\psi \phi}$ is allowed. We therefore see that the $Z^{\prime} \mathrm{FCNC}$ model is another type of NP which can explain the measured value of the $\mathrm{D} \varnothing$ dimuon charge asymmetry in the $B$ system.

Acknowledgments: We thank F. J. Botella for very helpful comments about the ZFCNC model. This work was financially supported by NSERC of Canada (AKA,DL). SB is grateful for the hospitality of the Université de Montréal, where part of this work was done. SB also acknowledges financial support from the Ba-

sic Science Research Program through the National Research Foundation of Korea (NRF), funded by the Ministry of Education, Science and Technology No. 20100028004.

\section{References}

[1] E. Lunghi and A. Soni, JHEP 0908, 051 (2009) [arXiv:0903.5059 [hep-ph]] and references therein.

[2] In the latest update of the $\pi K$ puzzle, it was seen that, although NP was hinted at in $B \rightarrow \pi K$ decays, it could be argued that the $\mathrm{SM}$ can explain the data, see S. Baek, C. W. Chiang and D. London, Phys. Lett. B 675, 59 (2009) [arXiv:0903.3086 [hep-ph]].

[3] A. Ishikawa et al. [Belle Collaboration], Phys. Rev. Lett. 96, 251801 (2006) [arXiv:hep-ex/0603018]; J. T. Wei et al. [BELLE Collaboration], Phys. Rev. Lett. 103, 171801 (2009) [arXiv:0904.0770 [hep-ex]]; B. Aubert et al. [BABAR Collaboration], Phys. Rev. D 73, 092001 (2006) [arXiv:hep-ex/0604007]; B. Aubert et al. [BABAR Collaboration], Phys. Rev. D 79, 031102 (2009) [arXiv:0804.4412 [hep-ex]]; A. K. Alok, A. Dighe, D. Ghosh, D. London, J. Matias, M. Nagashima and A. Szynkman, JHEP 1002, 053 (2010) [arXiv:0912.1382 [hep-ph]].

[4] M. Bona et al. [UTfit Collaboration], PMC Phys. A 3, 6 (2009) [arXiv:0803.0659 [hep-ph]].

[5] V. M. Abazov et al. [DØ Collaboration], arXiv:1005.2757 [hep-ex].

[6] A. Lenz and U. Nierste, JHEP 0706, 072 (2007) [arXiv:hep-ph/0612167]; A. Lenz, Nucl. Phys. Proc. Suppl. 177-178, 81 (2008) [arXiv:0705.3802 [hep-ph]].

[7] Z. Ligeti, M. Papucci, G. Perez and J. Zupan, arXiv:1006.0432 [hep-ph].

[8] E. Barberio et al. [Heavy Flavor Averaging Group], arXiv:0808.1297 [hep-ex].

[9] CDF Collaboration, Note 9015, Oct. 2007.

[10] V. M. Abazov et al. [DØ Collaboration], arXiv:0904.3907 [hep-ex].

[11] I. Dunietz, R. Fleischer and U. Nierste, Phys. Rev. D 63, 114015 (2001) [arXiv:hep-ph/0012219]. 
[12] N. G. Deshpande, X. G. He and G. Valencia, arXiv:1006.1682 [hep-ph].

[13] A. Dighe, A. Kundu and S. Nandi, arXiv:1005.4051 [hep-ph].

[14] A. Soni, A. K. Alok, A. Giri, R. Mohanta and S. Nandi, Phys. Rev. D 82, 033009 (2010) [arXiv:1002.0595 [hep-ph]]; C. H. Chen and G. Faisel, arXiv:1005.4582 [hep-ph]; C. W. Bauer and N. D. Dunn, arXiv:1006.1629 [hep-ph]; D. Choudhury and D. K. Ghosh, arXiv:1006.2171 [hep-ph]; C. H. Chen, C. Q. Geng and W. Wang, arXiv:1006.5216 [hep-ph]; J. K. Parry, arXiv:1006.5331 [hep-ph]; P. Ko and J. h. Park, arXiv:1006.5821 [hep-ph]; S. F. King, arXiv:1006.5895 [hep-ph]; C. Delaunay, O. Gedalia, S. J. Lee and G. Perez, arXiv:1007.0243 [hep-ph]; Y. Bai and A. E. Nelson, arXiv:1007.0596 [hep-ph]; J. Kubo and A. Lenz, arXiv:1007.0680 [hep-ph]; K. Blum, Y. Hochberg and Y. Nir, JHEP 1009, 035 (2010) [arXiv:1007.1872 [hep-ph]]; R. M. Wang, Y. G. Xu, M. L. Liu and B. Z. Li, arXiv:1007.2944 [hep-ph]; B. Dutta, Y. Mimura and Y. Santoso, arXiv:1007.3696 [hep-ph]; A. Lenz et al., arXiv:1008.1593 [hep-ph]; S. Oh and J. Tandean, arXiv:1008.2153 [hep-ph]; S. C. Park, J. Shu, K. Wang and T. T. Yanagida, arXiv:1008.4445 [hep-ph]; W. Chao and Y. c. Zhang, arXiv:1008.5277 [hep-ph]; M. Trott and M. B. Wise, arXiv:1009.2813 [hep-ph]; M. Blennow, B. Dasgupta, E. Fernandez-Martinez and N. Rius, arXiv:1009.3159 [hep-ph]; M. Endo, S. Shirai and T. T. Yanagida, arXiv:1009.3366 [hep-ph]; X. G. He, B. Ren and P. C. Xie, arXiv:1009.3398 [hep-ph].

[15] G. Buchalla, A. J. Buras and M. E. Lautenbacher, Rev. Mod. Phys. 68, 1125 (1996) [arXiv:hep-ph/9512380].

[16] D. Becirevic et al., Nucl. Phys. B 634, 105 (2002) [arXiv:hep-ph/0112303].

[17] J. Laiho, E. Lunghi and R. S. Van de Water, Phys. Rev. D 81, 034503 (2010) [arXiv:0910.2928 [hep-ph]].

[18] Y. Grossman, Z. Ligeti and E. Nardi, Phys. Rev. D 55, 2768 (1997) [arXiv:hep-ph/9607473].

[19] A. S. Dighe, I. Dunietz, H. J. Lipkin and J. L. Rosner, Phys. Lett. B 369, 144 (1996) [arXiv:hep-ph/9511363].

[20] A. S. Dighe, I. Dunietz and R. Fleischer, Eur. Phys. J. C 6, 647 (1999) [arXiv:hep-ph/9804253].

[21] T. Aaltonen et al. [CDF Collaboration], Phys. Rev. Lett. 100, 161802 (2008) [arXiv:0712.2397 [hep-ex]].

[22] V. M. Abazov et al. [D0 Collaboration], Phys. Rev. Lett. 101, 241801 (2008) [arXiv:0802.2255 [hep-ex]].

[23] C. W. Chiang, A. Datta, M. Duraisamy, D. London, M. Nagashima and A. Szynkman, JHEP 1004, 031 (2010) [arXiv:0910.2929 [hep-ph]]. 
[24] A. Datta and D. London, Phys. Lett. B 595, 453 (2004) [arXiv:hep-ph/0404130].

[25] Y. Grossman, Y. Nir and G. Perez, Phys. Rev. Lett. 103, 071602 (2009) [arXiv:0904.0305 [hep-ph]].

[26] For a review of ZFCNC models, see Y. Grossman, Y. Nir and R. Rattazzi, Adv. Ser. Direct. High Energy Phys. 15, 755 (1998) [arXiv:hep-ph/9701231], and references therein.

[27] G. Barenboim, F. J. Botella and O. Vives, Nucl. Phys. B 613, 285 (2001) [arXiv:hep-ph/0105306].

[28] G. Barenboim and F. J. Botella, Phys. Lett. B 433, 385 (1998) [arXiv:hep-ph/9708209].

[29] P. Langacker, Rev. Mod. Phys. 81, 1199 (2008) [arXiv:0801.1345 [hep-ph]].

[30] X. G. He and G. Valencia, Phys. Rev. D 74, 013011 (2006) [arXiv:hep-ph/0605202].

[31] S. Baek, J. H. Jeon and C. S. Kim, Phys. Lett. B 641, 183 (2006) [arXiv:hep-ph/0607113]; S. Baek, J. H. Jeon and C. S. Kim, Phys. Lett. B 664, 84 (2008) [arXiv:0803.0062 [hep-ph]].

[32] E. Golowich, S. Pakvasa and A. A. Petrov, Phys. Rev. Lett. 98, 181801 (2007) [arXiv:hep-ph/0610039].

[33] S. L. Chen, X. G. He, A. Hovhannisyan and H. C. Tsai, JHEP 0709, 044 (2007) [arXiv:0706.1100 [hep-ph]].

[34] A. K. Alok, A. Datta, A. Dighe, M. Duraisamy, D. Ghosh, D. London and S. U. Sankar, arXiv:1008.2367 [hep-ph].

[35] C. Bobeth, G. Hiller and G. Piranishvili, JHEP 0807, 106 (2008) [arXiv:0805.2525 [hep-ph]]; C. Bobeth, G. Hiller and D. van Dyk, JHEP 1007, 098 (2010) [arXiv:1006.5013 [hep-ph]].

[36] R. Mohanta and A. K. Giri, Phys. Rev. D 78, 116002 (2008) [arXiv:0812.1077 [hep-ph]].

[37] J. Charles et al. [CKMfitter Group], Eur. Phys. J. C 41, 1 (2005) [arXiv:hep-ph/0406184]. 